\title{
Video-observed therapy and medication adherence for tuberculosis patients: randomised controlled trial in Moldova
}

\author{
Luke Ravenscroft ${ }^{1}$, Stewart Kettle ${ }^{1}$, Ruth Persian ${ }^{1}$, Simon Ruda ${ }^{1}$, \\ Lilian Severin ${ }^{2}$, Svetlana Doltu ${ }^{2}$, Benjamin Schenck $\mathbb{1}^{3}$ and \\ George Loewenstein ${ }^{3}$
}

Affiliations: ${ }^{1}$ Behavioural Insights Team, London, UK. ${ }^{2}$ Act For Involvement, Chișinău, Moldova. ${ }^{3}$ Carnegie Mellon University, Pittsburgh, PA, USA.

Correspondence: Luke Ravenscroft, Behavioural Insights Team, 9 Raffles Place, Singapore 048619, Singapore. E-mail: luke.ravenscroft@bi.team

@ERSpublications

In this RCT, patients were assigned to either in-person observed treatment (as normal) or asynchronous video-observation. Observed medication adherence and satisfaction were improved and loss in patient time and costs during treatment were reduced. https://bit.ly/2Klmf18

Cite this article as: Ravenscroft L, Kettle S, Persian R, et al. Video-observed therapy and medication adherence for tuberculosis patients: randomised controlled trial in Moldova. Eur Respir J 2020; 56: 2000493 [https://doi.org/10.1183/13993003.00493-2020]

\section{ABSTRACT}

Introduction: The effectiveness of video-observed therapy (VOT) for treating tuberculosis (TB) has not been measured in low- and middle-income countries (LMICs), where $>95 \%$ of TB cases and deaths occur. In this study, we analyse the effectiveness and patient cost-difference of VOT compared to clinic-based directly observed therapy (DOT) in improving medication adherence in Moldova, a LMIC in Eastern Europe.

Methods: The study was a two-arm individually randomised trial with 197 TB patients ( $\mathrm{n}=99$ DOT control group; $\mathrm{n}=98$ VOT treatment group; multidrug-resistant TB cases were excluded). The primary outcome was observed medication adherence, measured by the number of days that a patient failed to be observed adhering to medication for every 2-week period during the course of their treatment.

Results: VOT significantly decreased nonadherence by 4 days (95\% CI 3.35-4.67 days, p<0.01) per 2-week period: 5.24 days missed per 2-week period for DOT and 1.29 days for VOT. VOT patients spent MDL 504 ( $\sim$ EUR 25) (95\% CI MDL 277-730, p<0.01) and $58 \mathrm{~h}(95 \% \mathrm{CI} 48-68 \mathrm{~h}, \mathrm{p}<0.01)$ less on their treatment. In addition, VOT increased self-reported satisfaction with treatment. We found no significant results pertaining to treatment success, patient wellbeing or patient employment status and some evidence of an increase in side-effects.

Discussion: In this trial, VOT increased observed medication adherence for TB patients in Moldova, a LMIC, when compared to clinic-based DOT. Additionally, VOT significantly reduced the time and money patients spent on their treatment.

This article has an editorial commentary: https://doi.org/10.1183/13993003.01998-2020

This article has supplementary material available from erj.ersjournals.com

This study was registered at ClinicalTrials.gov with identifier NCT02331732. The study protocol, informed consent and statistical analysis plan can be shared. Data can be provided to researchers that provide a methodologically sound proposal. Aggregate data for all of the outcomes we collected in our trial can also be shared. For individual de-identified participant data, we will need to seek permission from our Moldovan partners. The data can be made available for sharing beginning 3 months and ending 36 months following journal publication.

Received: 12 Sept 2019 | Accepted after revision: 13 April 2020

Copyright $\odot$ ERS 2020 


\section{Introduction}

Among medical conditions requiring adherence to treatment regimens, adherence to tuberculosis (TB) medication is particularly important, due to risks of transmission. Furthermore, patients who fail to follow the recommended treatment closely are more likely to develop drug-resistant forms of $\mathrm{TB}$, which further complicates the treatment process [1]. TB poses special challenges when it comes to adherence, for several reasons, including the absence of immediately observable benefits, time lags between administration and impact and the long duration of anti-TB regimens [2]. In addition, TB patients tend to be from lower education and income groups, characteristics that are generally associated with lower levels of adherence [3]. In combination, all of these factors help to explain why TB treatment in many countries is legally mandated and monitored via in-person visits to clinics, as is the practice in Moldova.

In 1993, the World Health Organization (WHO) recommended "effective case management via direct observation of treatment (DOT) by an independent and trained third party" as a response to decades of reports documenting the failure of patients to complete treatment [4]. Since the WHO DOT recommendations were provided, two Cochrane reviews $[5,6]$ found no evidence that DOT was more effective than self-administered treatment. However, with the advent and proliferation of mobile devices equipped with internet and video capabilities, a new approach to treatment has been developed: video-observed therapy (VOT), whereby observation is conducted remotely. In a VOT procedure, patients use mobile devices and a secure application for recording and sending videos to case workers, who view and document each event [7]. VOT can be synchronous (S-VOT), where patients and providers engage in a live videoconference, or asynchronous (A-VOT), where patients upload the videos to a secure content management system that can be accessed by the provider [8].

In 2015, the WHO Global TB Programme established the global task force on digital health in partnership with the European Respiratory Society to support the development of digital health innovations [9]. The agenda, which supports the WHO's End TB strategy, advocates that TB programmes use digital health solutions, such as VOT, in their implementation, and that they invest in research to measure the effectiveness of digital health interventions [10-12]. VOT was one of nine products identified by the Task Force for a Target Product Profile, a strategic document that specifies the features of an information and communication technology product [13].

The most comprehensive study to date which evaluates VOT versus DOT in an experimental setting was a randomised controlled superiority trial in London (UK) [14]. Similar to our study, the participants were randomised into VOT and DOT, and researchers found a substantial increase in observed adherence. With the vast majority of TB cases occurring in developing nations, the authors made a call for "more research in this area, including comparative studies between different digital adherence interventions in high-burden settings...”. A 2018 review on the impact of digital health technologies on tuberculosis treatment by NGWATU et al. [15] made a similar call for more studies of better quality: “...further evaluation of digital health interventions is urgently needed - ideally in adequately powered RCTs [randomised controlled trials]...”. Finally, a review by NsEngrYumva et al. [16] on the costs and impact of different digital health technologies for TB treatment found support for VOT reducing patient costs in a high-burden country, Brazil, by using decision analysis model simulations. Our aim in this study is to start to fill this research gap by rigorously evaluating the effectiveness and cost-differences of VOT for non-multidrug-resistant (MDR)-TB patients in a low- and middle-income country (LMIC) with a high disease burden.

In 2015, at 152 cases per 100000 population, Moldova had the highest incidence of TB in Europe [17]. Moldova follows a clinic-based strategy of DOT. Interviews with patients and discussions with the Ministry of Health suggested that the current implementation of DOT was challenging for some patients, providing a case for testing out a potentially more convenient approach to monitoring adherence. Under DOT, TB patients in Moldova are required by law to come to a clinic daily to take their medication under the supervision of a TB nurse. To date, there has not been a robust evaluation of the efficacy of VOT compared to DOT in a LMIC, despite $>95 \%$ of all deaths caused by TB occurring in these countries [18].

\section{Methods}

\section{Study design}

We conducted the RCT in Chişinău, the capital of Moldova, because of its high rate of internet penetration compared to the rest of the country at the time the trial was designed. The RCT ran for 22 months, from January 2016 to November 2017, when the 4-month monitoring period for the last patient recruited into the trial was completed.

The study was a two-arm individually randomised clinical trial with a parallel design. Patients were approached just before the beginning of their treatment continuation phase. In order to be eligible to 
participate, patients needed to consent to taking part in the trial and meet the following criteria. 1) Resident in Chişinău with no plans to move away from Chişinău during the 4 months of the trial; 2) age $\geqslant 18$ years; 3 ) have $\geqslant 4$ months of treatment remaining; 4) do not have MDR-TB; 5) are not homeless; 6) do not suffer from alcoholism or drug misuse; 7) are not in prison; and 8) are in either category I (intensive phase and/or continuation phase) or category II (continuation phase or phase after finishing intensive intramuscular injection of streptomycin) phase of treatment. Patients did not need to be proficient with mobile phones, tablets or mobile applications to be included in the study.

Recruitment occurred on a rolling basis from the list of patients declared by the municipal coordinator. These cases were verified by doctors, then visited in the hospital or at their first visits to an outpatient unit. Patients completed the baseline questionnaire (appendix 1), and those who met the eligibility requirements were provided with verbal and written information on the trial and the two treatment types. Immediately following this, patients were given the option to participate in the trial. If they agreed and provided written consent to participate, they were immediately randomised to DOT or VOT using a custom-made online randomisation tool. Patients then started DOT or VOT at the very beginning of their continuation phase of treatment. Given the rolling nature of recruitment, simple randomisation (i.e. no blocking) was used, and given the tangible difference between the treatments, it was not feasible to blind patients to their assignment. VOT patients who continued their treatment beyond 4 months continued VOT to the end of their treatment. In the event a patient in VOT was hospitalised, they would continue VOT once they left hospital.

Our power analysis indicated that 188 patients would allow us to see a 1.5 -day shift in observed adherence between groups. Therefore, our aim was to recruit a sample of $\sim 200$ patients. For treatment success, this sample size would allow for the detection of a difference of $9.9 \%$ in treatment success at a 12 -month cut-off. Of the 197 patients who entered the trial, 99 were assigned to the control group and received the standard provision of DOT from their local clinic (one of the 15 clinics in Chişinău), and 98 were assigned to VOT.

All patients were incentivised to adhere to their treatment using food vouchers worth MDL 980 ( $\sim$ EUR 50) a month, as was standard practice for DOT TB treatment at the time. Both DOT and VOT patients received their food voucher on a weekly or monthly basis if they adhered $\geqslant 90 \%$ of the time.

We received approval from the Moldovan Ministry of Health to conduct the trial. The Ministry of Health, Labour and Social Protection in Moldova relaxed current TB treatment guidelines to enable implementation of the trial. In addition, ethical clearance was received from the Moldovan ethics board and the University College London (UCL) ethics board.

\section{Procedure: DOT}

The patients assigned to DOT underwent the procedure that has been used in Moldova for $>10$ years: they were required to go to their local TB clinic daily (Monday to Friday) and be observed taking their medicine. During this visit, patients would also be asked to report any side-effects. Under the official guidelines, patients in the DOT arm can only take their medication between Monday and Friday at their local clinic. However, in practice, patients are sometimes given additional medications to take at home if they know they are going to miss a subsequent DOT visit, in which case the patient would be automatically marked as adhering for the subsequent (missed) visit(s). Given this occasional informal arrangement, to ensure the accuracy of the adherence outcome, we implemented a new monitoring procedure for DOT patients. In addition to marking attendance on paper as normal, patients were required to sign a tablet which recorded date and time, removing the possibility that patients could be marked as adhering for multiple days in one visit, as described. This provided an accurate measure of observed adherence in the control group.

All DOT patients were assigned to one of 15 clinics, all in the capital Chişinău, based on proximity to their home address. For DOT patients, the nurse would mark their attendance on a paper "TB-01" form and the patient would sign. In addition, the patient would be asked to electronically sign-in on a tablet. Then the nurse would give the patient their medication, and observe them taking it. If the patient did not turn up for more than two consecutive days, the clinic would try and call the patient to encourage them to come back to the clinic.

\section{Procedure: VOT}

The patients assigned to VOT underwent training to understand all steps involved in performing asynchronous VOT. First, they visited an observation centre and were given a VOT medication sheet (appendix 2), VOT video recording procedure (appendix 3) and detailed instructions of how to show that they swallowed their medication. Each time patients sent a video, they were asked to report side-effects. 
Finally, an mHealth app, designed for the trial, was installed on any internet-enabled devices that they owned. If they didn't own one, they were loaned a tablet with the mHealth app already installed for the remainder of the trial.

VOT patients were assigned to a clinic where they received a 14-day supply of medicine. Patients were instructed to send a video daily of themselves swallowing the medication to the VOT observer, who determined if they could clearly see the patient taking the medicine. After each video, the observer would respond with a video confirming receipt of the clip and encouraging patients to keep taking their medicine as a way to provide personalised patient-provider interaction, one of the main advantages of synchronous VOT and DOT [8]. If a VOT patient missed sending a video, the observer would phone the patient to find out if there were any problems and encourage the patient to submit a video on the following day. These reminders and video feedback aimed to provide the practical and emotional social support that has been found to be associated with higher medication adherence [19].

The VOT observers were certified medical assistants, with basic knowledge about TB. Additionally, we conducted training with the observers on database data entry, communication with patients (video feedback and reminders in case of missing doses), distribution of drugs and management of side-effects.

\section{Sample}

Out of 197 eligible participants, 99 were randomised to the DOT control group and 98 to the VOT treatment group. After randomisation, 13 participants (five in DOT, eight in VOT) were excluded for medical reasons, such as developing MDR-TB, and six (one in DOT, five in VOT) refused to participate at either the beginning of the trial or later. In addition, one patient (randomised to DOT) died, and two (randomised to DOT) were lost to follow-up. The only differences that were statistically significant were a higher rate of loss to follow-up in the DOT condition (significant at the 5\% level) and a greater initial refusal of participation in the VOT treatment condition (significant at the $10 \%$ level), but the numbers of both were small and are unlikely to have had much of an impact on results. Adherence analysis was conducted on the remaining 178 patients in the sample, with 93 in DOT and 85 in VOT (figure 1).

There were no statistically significant differences between age, sex, employment status, having drug or alcohol abuse problems, being homeless or imprisoned in the past 5 years, suggesting that our control and treatment groups were balanced across these demographic characteristics (tables 1 and 2). Balance was observed on most clinical characteristics (measured at baseline), apart from history of nonadherence, having experienced fatigue (both differences significant at the $10 \%$ level) and satisfaction with treatment at baseline (which we controlled for in our analysis).

\section{Outcomes and analysis}

Our primary outcome, adherence to medication, is the number of days over each 2-week period (10 working days, excluding weekends and public holidays) that a patient was not observed adhering to their medication. For DOT patients, this was based on whether they electronically signed the tablet at their clinic to indicate their attendance. For VOT patients, this was based on whether they sent a video showing them taking their medication. Across the monitoring period, each patient contributed approximately eight 2-week periods.

In addition, we recorded several secondary outcome measures. On a daily basis, patients were asked to record any side-effects (at the clinic for DOT and on the video for VOT). After 4 months, patients received an endline questionnaire (appendix 4). These self-reported questions provide the secondary outcomes for the time and money spent on their treatment, satisfaction with treatment, employment status and wellbeing (measured using a short-form of the Warwick-Edinburgh Mental Wellbeing Scale) [20]. Treatment success was measured by sputum smear and radiography, according to the national TB protocol and WHO guidelines at 4, 10 and 12 months after the start of the continuation phase [21].

All continuous outcome measures were evaluated with ordinary least-squares multiple regression using data from up to a 4-month period following randomisation into the trial (table 3). All binary outcome measures were evaluated with a logistic regression (table 3). Statistical analysis was conducted by Stewart Kettle and Ruth Persian. Neither was blinded to the trial arm while conducting the analysis.

\section{Results}

Primary outcome: adherence

In the DOT control group, patients failed to adhere 5.24 days per 2-week period. The VOT treatment significantly decreased nonadherence to 1.29 days per 2-week period, i.e. by nearly 4 days (95\% CI 3.354.67 days, $\mathrm{p}<0.01$; standard errors are clustered by patient to account for multiple observations per individual). The only other statistically significant characteristic was drug abuse problems at baseline. 


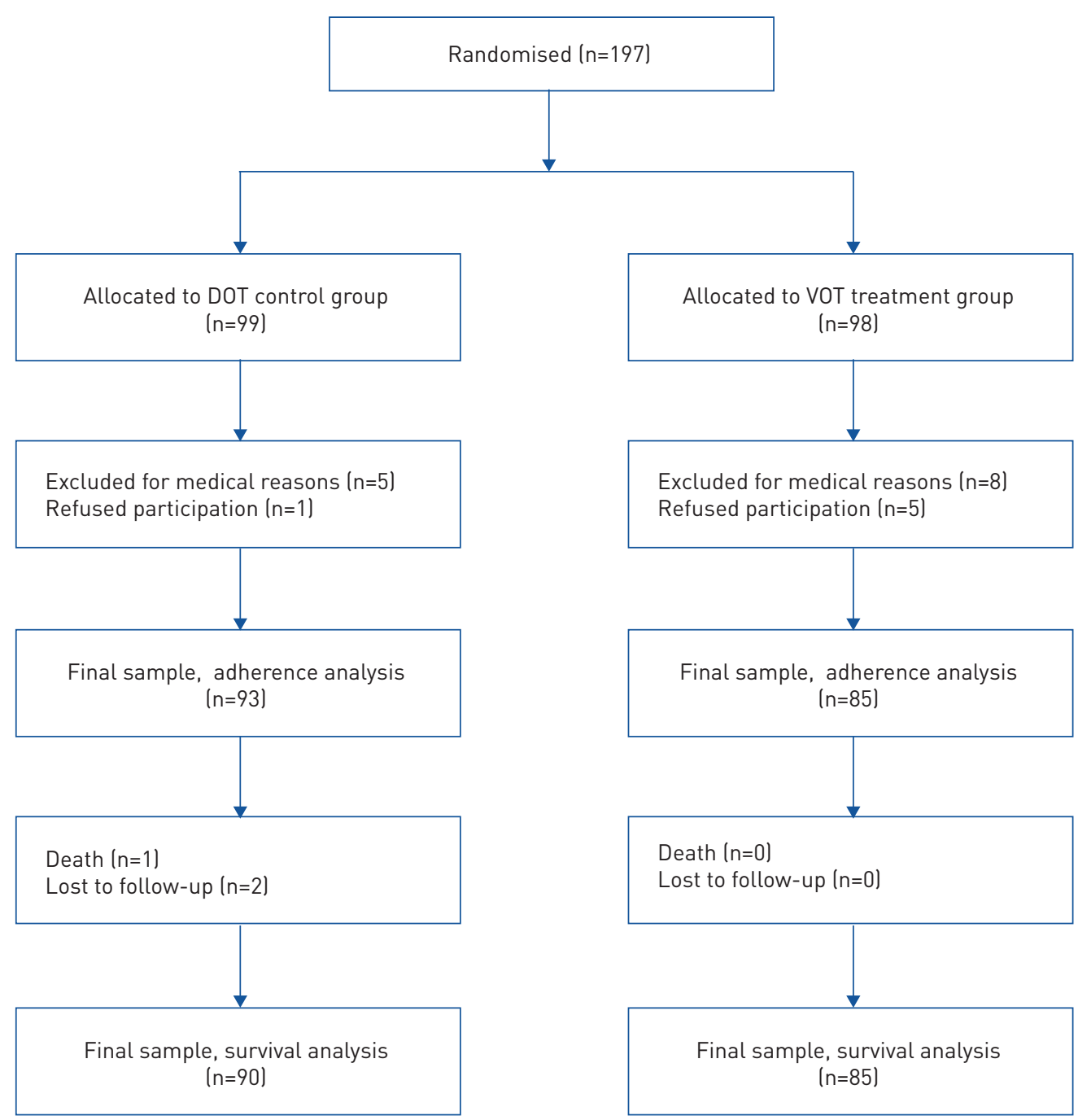

FIGURE 1 Consort flow diagram. DOT: directly observed treatment; VOT: video-observed treatment.

However, there were only two VOT patients reporting drug abuse problems and none in DOT, so this result should be interpreted with caution. As a robustness check, we performed the main regression analysis (table 3, column 1), but included history of nonadherence and satisfaction with treatment at baseline (significantly different at the $10 \%$ and $5 \%$ levels at baseline, respectively, as shown in table 2). The results did not change meaningfully: the coefficient of VOT is $3.764 \pm 0.354(\mathrm{p}<0.01)$ instead of $4.009 \pm 0.335(\mathrm{p}<0.01)$.

\begin{tabular}{lccc}
\multicolumn{2}{l}{ TABLE 1 Summary of baseline characteristics (demographic) } & & \\
& DOT & VOT & \\
\hline Subjects & 93 & 85 & 0.831 \\
Age years & $38.28 \pm 14.11$ & $38.73 \pm 13.95$ & 0.164 \\
Employed at baseline (binary) & $0.33 \pm 0.47$ & $0.44 \pm 0.50$ & 0.138 \\
Drug abuse problem (binary) & $0.00 \pm 0.00$ & $0.02 \pm 0.15$ & 0.272 \\
Alcohol abuse problem (binary) & $0.01 \pm 0.10$ & $0.04 \pm 0.19$ & 0.297 \\
Homeless at baseline (binary) & $0.00 \pm 0.00$ & $0.01 \pm 0.11$ & 0.297 \\
Imprisoned in past 5 years (binary) & $0.00 \pm 0.00$ & $0.01 \pm 0.11$ & 0.952 \\
Female (binary) & $0.45 \pm 0.50$ & $0.45 \pm 0.50$ & \\
\hline
\end{tabular}

Data are presented as $\mathrm{n}$ or mean \pm SD, unless otherwise stated. DOT: directly observed treatment; VOT: video-observed treatment. " : measured at beginning of continuation period, i.e. before randomisation; $\eta_{\text {: }} \mathrm{t}$-tests of the differences of the means. 


\begin{tabular}{|c|c|c|c|}
\hline & DOT & VOT & p-values $\rrbracket$ \\
\hline Height $\mathrm{cm}$ & $169.55 \pm 8.36$ & $170.82 \pm 8.42$ & 0.312 \\
\hline Weight kg & $64.13 \pm 12.89$ & $66.60 \pm 10.77$ & 0.169 \\
\hline Smear positive (binary) & $0.19 \pm 0.40$ & $0.21 \pm 0.41$ & 0.764 \\
\hline Culture positive (binary) & $0.33 \pm 0.47$ & $0.35 \pm 0.48$ & 0.785 \\
\hline History of nonadherence (binary) & $0.04 \pm 0.20$ & $0.00 \pm 0.00$ & $0.054^{+}$ \\
\hline \multicolumn{4}{|l|}{ Location of TB } \\
\hline Pulmonary (binary) & $0.95 \pm 0.23$ & $0.92 \pm 0.28$ & 0.450 \\
\hline Miliary (binary) & $0.01 \pm 0.10$ & $0.00 \pm 0.00$ & 0.340 \\
\hline Spinal (binary) & $0.00 \pm 0.00$ & $0.02 \pm 0.15$ & 0.138 \\
\hline Osteoarticular (binary) & $0.01 \pm 0.10$ & $0.01 \pm 0.11$ & 0.949 \\
\hline \multicolumn{4}{|l|}{ Sensitivity } \\
\hline Totally sensitive (binary) & $0.8 \pm 80.33$ & $0.84 \pm 0.37$ & 0.682 \\
\hline Isoniazid resistant (binary) & $0.01 \pm 0.10$ & $0.02 \pm 0.15$ & 0.511 \\
\hline Rifampicin resistant (binary) & $0.01 \pm 0.10$ & $0.00 \pm 0.00$ & 0.340 \\
\hline Ethambutol resistant (binary) & $0.00 \pm 0.00$ & $0.00 \pm 0.00$ & \\
\hline Pyrazinamide resistant (binary) & $0.00 \pm 0.00$ & $0.00 \pm 0.00$ & \\
\hline \multicolumn{4}{|l|}{ Side-effects } \\
\hline Nausea, vomiting (binary) & $0.09 \pm 0.28$ & $0.05 \pm 0.21$ & 0.303 \\
\hline Stomach pain (binary) & $0.09 \pm 0.28$ & $0.05 \pm 0.21$ & 0.303 \\
\hline Fatigue (binary) & $0.10 \pm 0.30$ & $0.20 \pm 0.40$ & $0.052^{+}$ \\
\hline Fever (binary) & $0.01 \pm 0.10$ & $0.00 \pm 0.00$ & 0.340 \\
\hline Rash, severe itching (binary) & $0.02 \pm 0.15$ & $0.04 \pm 0.19$ & 0.581 \\
\hline Paraesthesiae (binary) & $0.01 \pm 0.10$ & $0.01 \pm 0.11$ & 0.949 \\
\hline Vertigo (binary) & $0.00 \pm 0.00$ & $0.00 \pm 0.00$ & \\
\hline Jaundice (binary) & $0.00 \pm 0.00$ & $0.01 \pm 0.11$ & 0.297 \\
\hline Arthralgia (binary) & $0.02 \pm 0.15$ & $0.02 \pm 0.15$ & 0.928 \\
\hline Loss of appetite (binary) & $0.03 \pm 0.18$ & $0.06 \pm 0.24$ & 0.396 \\
\hline Any side-effects (binary) & $0.20 \pm 0.41$ & $0.31 \pm 0.46$ & 0.121 \\
\hline Satisfied with treatment (binary) & $0.82 \pm 0.39$ & $0.97 \pm 0.19$ & $0.002^{* *}$ \\
\hline Self-rated health (scale $0-100$ ) & $89.09 \pm 10.76$ & $89.80 \pm 8.72$ & 0.629 \\
\hline
\end{tabular}

Data are presented as mean \pm SD, unless otherwise stated. DOT: directly observed treatment; VOT: video-observed treatment; TB: tuberculosis. "\#: measured at beginning of continuation period, i.e. before randomisation; ${ }^{\text {ๆ }}$ : t-tests of the differences of the means. ${ }^{+}: p<0.1 ;{ }^{* *}: p<0.01$.

\section{Secondary outcomes}

We observed an encouraging and statistically significant impact on whether a patient achieved $80 \%$ adherence in any given 2-week period, a canonical threshold used to measure "good" adherence [22]. DOT patients achieved $80 \%$ medication adherence $19.5 \%$ of the time. The VOT treatment significantly increased patients' likelihood of meeting this threshold to $75.1 \%$, or by $55.6 \%$ (95\% CI $48-63 \%$; p $<0.01$ ) (figure 2).

Patients in the VOT condition saved an average of $58 \mathrm{~h}$ (95\% CI 48-68 h; p<0.01) over the course of the 4 -month period. This was calculated from self-reported time spent on treatment. In addition, patients in the VOT condition saved an average of MDL 504 (95\% CI MDL 277-730; $<<0.01$ ), or EUR 25 over the course of the 4-month period, again based on self-reports.

Patients in the VOT condition reported greater satisfaction with their treatment. Controlling for satisfaction at baseline, the cumulative log-odds increment in satisfaction of being in the treatment group is 3.29 (95\% CI $1.66-4.92 ; \mathrm{p}<0.01)$. A regression of binary and five-point treatment satisfaction outcomes controlling for satisfaction at baseline is included in table S4.

Measuring treatment success by sputum smear and radiography, as according to the national TB protocol and WHO guidelines [21], we did not find a significant impact of treatment allocation on treatment success at 4 months after starting the continuation phase (DOT 15.0\%, VOT 11.1\%), 10 months (DOT 86.0\%, VOT 92.1\%) or 12 months (DOT 90.3\%, VOT 93.5\%). Our sample size allowed for the detection of a difference of $9.9 \%$ for the 12 -month cut-off; to detect a $5 \%$ increase with $5 \%$ statistical significance and $80 \%$ power would have required a sample of 976 individuals.

We observed no statistically significant difference in patient wellbeing or patient employment status between DOT and VOT patients. Finally, patients in the VOT condition are 11\% (95\% CI -1.9-24.31\%; $\mathrm{p}<0.10)$ more likely to report side-effects in the endline questionnaire than in the DOT condition. 


\begin{tabular}{|c|c|c|c|c|c|c|c|c|c|}
\hline & $\begin{array}{c}\text { Nonadherence } \\
\text { events per } \\
10 \text { days } \#\end{array}$ & $\begin{array}{c}\text { 12-month } \\
\text { treatment } \\
\text { success }\end{array}$ & $\begin{array}{c}80 \% \\
\text { adherence }\end{array}$ & $\begin{array}{c}\text { Wellbeing } \\
\text { (WEMWBS5) }^{+}\end{array}$ & $\begin{array}{c}\text { Patient } \\
\text { satisfaction }\end{array}$ & Time spent $\mathbf{h}^{+}$ & Money spent $\mathrm{MDL}^{+}$ & Employed" & $\begin{array}{c}\text { Any } \\
\text { side-effects }\end{array}$ \\
\hline VoT & $-4.009 \pm 0.335^{* *}$ & $1.548 \pm 0.901$ & $12.795 \pm 2.738^{* *}$ & $-0.520 \pm 0.419$ & $11.879 \pm 14.262 *$ & $-\mathbf{5 8 . 0 5 8} \pm 5.122^{* *}$ & 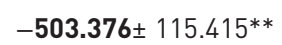 & $1.479 \pm 0.502$ & $1.882 \pm 0.711^{\S}$ \\
\hline Female & $-0.087 \pm 0.347$ & $1.658 \pm 0.977$ & $0.948 \pm 0.201$ & $-0.147 \pm 0.420$ & $0.688 \pm 0.644$ & $-1.042+5.434$ & $-117.491 \pm 127.890$ & $0.583 \pm 0.204$ & $1.668 \pm 0.616$ \\
\hline Age years & $-0.007 \pm 0.012$ & $1.015 \pm 0.023$ & $0.991 \pm 0.007$ & $-0.012 \pm 0.016$ & $1.021 \pm 0.031$ & $-0.103 \pm 0.173$ & $-8.062 \pm 4.244^{\S}$ & $0.979 \pm 0.013^{\S}$ & $1.026 \pm 0.012 *$ \\
\hline $\begin{array}{l}\text { Employed at } \\
\text { baseline }\end{array}$ & $-0.171 \pm 0.346$ & $1.516 \pm 0.925$ & $1.190 \pm 0.254$ & $-0.232 \pm 0.421$ & $3.643 \pm 3.234$ & $8.617 \pm 5.263$ & $12.020 \pm 122.227$ & $3.296 \pm 1.144^{* *}$ & $0.546 \pm 0.215$ \\
\hline $\begin{array}{l}\text { Drug misuse } \\
\text { problem at } \\
\text { baseline }\end{array}$ & $3.632 \pm 0.310^{* *}$ & & $0.293 \pm 0.065^{* *}$ & $-6.242 \pm 0.448^{* *}$ & & $-6.558 \pm 4.643$ & $-142.430 \pm 74.172^{\S}$ & & \\
\hline $\begin{array}{l}\text { Alcohol misuse } \\
\text { problem at } \\
\text { baseline }\end{array}$ & $0.377 \pm 1.148$ & & $1.153 \pm 0.716$ & $-2.254 \pm 2.921$ & & $-8.632 \pm 6.818$ & $-250.613 \pm 123.216^{*}$ & $0.848 \pm 1.148$ & \\
\hline $\begin{array}{l}\text { Homeless at } \\
\text { baseline }\end{array}$ & $-1.097 \pm 1.258$ & & $0.396 \pm 0.279$ & $11.299 \pm 2.999 * *$ & & $2.175 \pm 9.487$ & $214.223 \pm 195.636$ & & \\
\hline $\begin{array}{c}=1 \text { if satisfied at } \\
\text { baseline } \\
\text { (binary) }\end{array}$ & & & & & $239.180 \pm 229.852^{* *}$ & & & & \\
\hline $\begin{array}{l}\text { Control group } \\
\text { mean }\end{array}$ & 5.240 & 0.903 & 0.019 & 22.697 & 0.820 & 80.865 & 696.800 & 0.333 & 0.211 \\
\hline Observations $\mathrm{n}$ & 1571 & 173 & 1571 & 172 & 167 & 163 & 155 & 169 & 170 \\
\hline
\end{tabular}



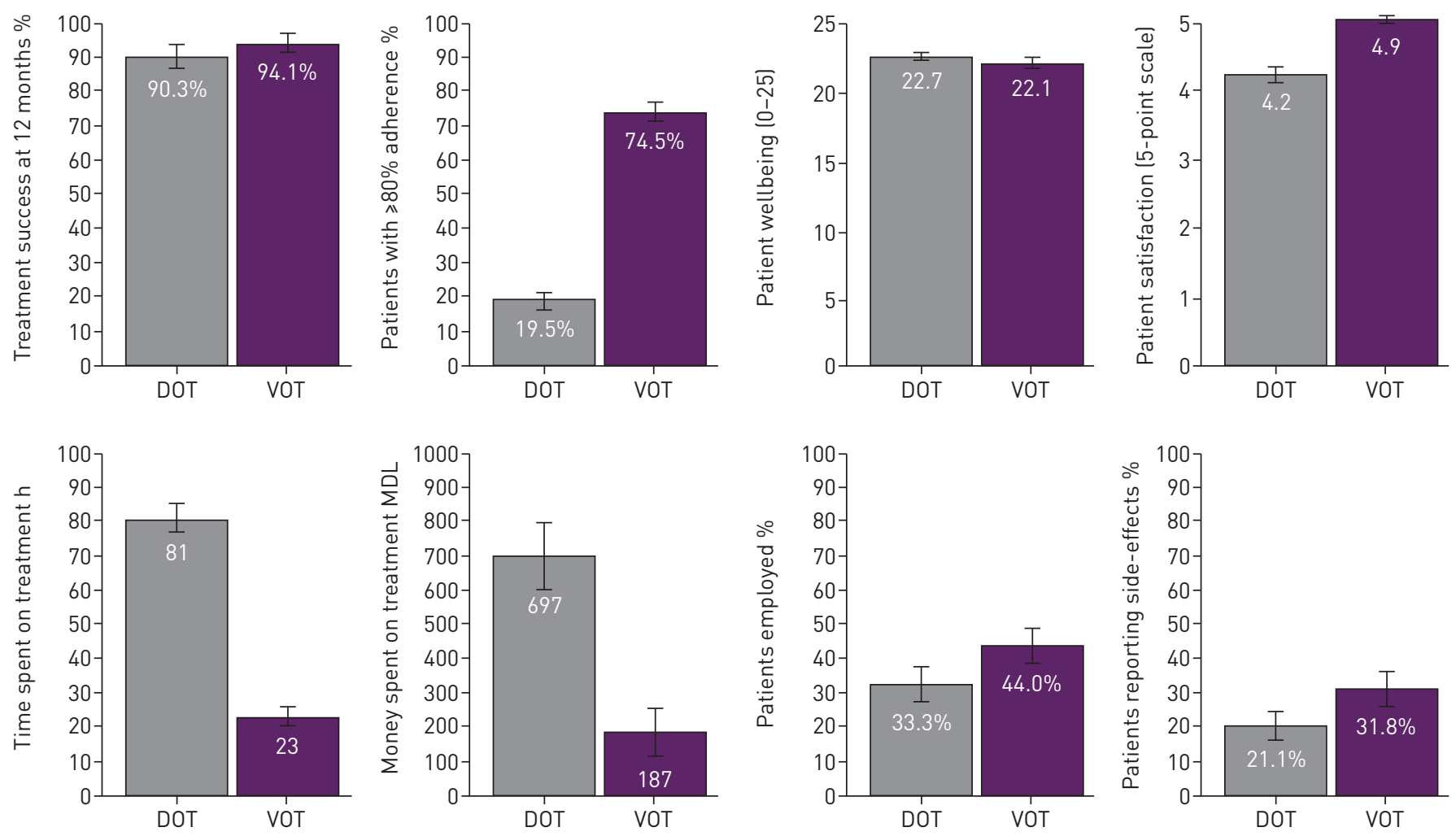

FIGURE 2 Summary of secondary outcomes. Numbers are the average of continuous variables; percentages are averages of binary variables. Data are presented as mean \pm SE. DOT: directly observed treatment; VOT: video-observed treatment; MDL: Moldovan leu.

\section{Discussion}

In this trial, VOT increased observed medication adherence for TB patients compared to clinic-based DOT, a difference of 4 days of adherence per 2-week period. In addition, VOT saved patients time and money and increased their satisfaction, which can all be seen as explanations for higher adherence and as benefits of VOT over DOT. The study demonstrates both the feasibility of using VOT on challenging medication adherence problems in LMICs, and the substantial time and monetary savings that result from doing so. The monetary savings are likely to be an underestimate, as the endline questionnaire (appendix 4) only asked for self-reported expenditures on transportation, not foregone wages and the increase of child/dependent care resulting from in-person treatment.

The $11 \%$ increase in reported side-effects is important and worth discussing. The increase in reported side-effects should not be seen as a drawback of the study. It could simply be that more side-effects were reported by VOT patients because more medicine was taken. It could also be that the new approach to and training on reporting side-effects (asking VOT patients to report any side-effects every day when submitting the video) encouraged more regular reporting of side-effects compared with the approach adopted by the TB nurse at the clinic. This would also be a positive result as it is important to identify side-effects, as medical attention or a change in treatment may be required. While our study doesn't capture these measures, this potential improvement in quality of care is one of the advantages of digital adherence technologies.

The implications of the study are important, but there are also some important limitations. The first is that our primary outcome measure (observed adherence) only measures whether we observe the patient adhering, not whether the patient actually adheres. The difference in observed adherence could overestimate the true difference in adherence if patients in the DOT condition took their medication when they did not go to the clinic, and could be an underestimate if patients in the VOT condition took their medication without sending the video. However, a key finding is that measured adherence in the VOT condition was very high: $75 \%$ of VOT patients took $\geqslant 80 \%$ of their medicine. This could underestimate the true adherence, but is very unlikely to be an overestimate, given that each episode of adherence was confirmed by video by a trained observer.

The second main limitation is the sample size. When evaluating the impact on treatment success, although there was directional improvement in treatment success at 10- and 12-month cut-offs, the findings are 
nonsignificant. We would have needed 976 patients in our study to detect a $5 \%$ increase in treatment success after a year, which was beyond the scope and resources of the study. However, it is still important to consider, given the large improvement in observed adherence, why we didn't see a larger change in treatment success. It could be that our measure of observed adherence overestimates the improvement in actual adherence due to VOT. Alternatively, given that the minimum rate at which TB medication has to be taken to be effective is unknown, it might be that while DOT patients showed poorer adherence than VOT, this was sufficient to reach the threshold for treatment success.

A third limitation of the study is the generalisability of the findings to regions where internet is less accessible because the VOT arm required patients to have internet access to upload the videos. However, as internet and smartphone penetration increase in LMICs, VOT should become an increasingly viable option for TB treatment.

Despite these limitations, the implications of this study are important. This is the first study that measures the difference between DOT and VOT treatment strategies in a LMIC. As connectivity to remote areas and voice/video quality improves, VOT will more closely emulate the patient-provider interaction, one of the benefits of DOT. Our findings not only confirm that VOT is more effective and has lower cost than DOT, but also provides evidence that these benefits are achievable in LMICs where $>95 \%$ of TB cases and deaths are observed.

Acknowledgements: This article is based on a project initiated jointly with UNDP Moldova. Michael Sanders (Behavioural Insights Team, London, UK) provided invaluable advice on evaluation design and analysis. Alex Tupper, David Nolan, Eskil Forsell, Chris Larkin and Flo Farghly (Behavioural Insights Team) designed the data collection tool and advised on the evaluation and monitoring of the trial. In addition, we would like to thank Andrew Hayward, Alistair Story, Rob Aldridge and Fatima Wurie (University College London, London, UK) and Sara Hemming (Royal Free London NHS Foundation Trust, London) for their advice on the trial design and VOT implementation. The Moldova National TB Programme helped with data analysis, evaluation and validation.

Conflict of interest: L. Ravenscroft reports consultancy fees from United Nations Development Programme (UNDP), during the conduct of the study. S. Kettle reports consultancy fees from United Nations Development Programme (UNDP), during the conduct of the study. R. Persian reports consultancy fees from United Nations Development Programme (UNDP), during the conduct of the study. S. Ruda reports consultancy fees from United Nations Development Programme (UNDP), during the conduct of the study. L. Severin reports personal fees from UNDP Moldova, grants from PAS Center, non-financial support from Moldcell Company, during the conduct of the study; personal fees from UNDP Moldova, outside the submitted work. S. Doltu reports personal fees from UNDP Moldova, grants from PAS Center, non-financial support from Moldcell Company, during the conduct of the study; personal fees from UNDP Moldova, outside the submitted work. B. Schenck has nothing to disclose. G. Loewenstein has nothing to disclose.

Support statement: The Behavioural Insights Team received funding for their time spent on the project from the United Nations Development Programme. In addition, Moldcell provided unlimited internet traffic for patients in the VOT arm free of charge. No financial support was provided to any individuals for the project. Funding information for this article has been deposited with the Crossref Funder Registry.

\section{References}

1 Centers for Disease Control and Prevention. Treatment for TB Disease. US Department of Health and Human Services, 2016. www.cdc.gov/tb/topic/treatment/tbdisease.htm Date last accessed: April 1, 2020.

2 Osterberg L, Blaschke T. Adherence to medication. N Engl J Med 2005; 353: 487-497.

3 Rolnick SJ, Pawloski PA, Hedblom BD, et al. Patient characteristics associated with medication adherence. Clin Med Res 2013; 11: 54-65.

4 Frieden TR, Sbarbaro JA. Promoting adherence to treatment for tuberculosis: the importance of direct observation. Bull World Health Organ 2007; 85: 407-409.

5 Volmink J, Garner P. Directly observed therapy for treating tuberculosis. Cochrane Database Syst Rev 2007; 4: CD003343.

6 Karumbi J, Garner P. Directly observed therapy for treating tuberculosis. Cochrane Database Syst Rev 2015; 5: CD003343.

7 Garfein RS, Collins K, Muñoz F, et al. Feasibility of tuberculosis treatment monitoring by video directly observed therapy: a binational pilot study. Int J Tuberc Lung Dis 2015; 19: 1057-1064.

8 Garfein RS, Doshi R. Synchronous and asynchronous video observed therapy (VOT) for tuberculosis treatment adherence monitoring and support. J Clin Tuberc Other Mycobact Dis 2019; 17: 100098.

9 World Health Organization. Digital Health for the End TB Strategy: an Agenda for Action (No. WHO/HTM/TB/ 2015.21). Geneva, World Health Organization, 2015.

10 Falzon D, Raviglione M, Bel EH, et al. The role of eHealth and mHealth in tuberculosis and tobacco control: a WHO/ERS consultation. Eur Respir J 2015; 46: 307-311.

11 Falzon D, Timimi H, Kurosinski P, et al. Digital health for the End TB Strategy: developing priority products and making them work. Eur Respir J 2016; 48: 29-45.

12 World Health Organization. Digital Health for the End TB Strategy: Progress Since 2015 and Future Perspectives. Meeting Report, 7-8 February 2017. www.who.int/tb/publications/digitalhealth-meetingreport2017/en/

13 World Health Organization. Target Product Profiles and Priority Digital Health Products for TB. 2015. www.who. int/tb/areas-of-work/digital-health/target-product-profiles/en/ 
14 Story A, Aldridge RW, Smith CM, et al. Smartphone-enabled video-observed versus directly observed treatment for tuberculosis: a multicentre, analyst-blinded, randomised, controlled superiority trial. Lancet 2019; 393: $1216-1224$

15 Ngwatu BK, Nsengiyumva NP, Oxlade O, et al. The impact of digital health technologies on tuberculosis treatment: a systematic review. Eur Respir J 2018; 51: 1701596.

16 Nsengiyumva NP, Mappin-Kasirer B, Oxlade O, et al. Evaluating the potential costs and impact of digital health technologies for tuberculosis treatment support. Eur Respir J 2018; 52: 1801363.

17 European Centre for Disease Prevention and Control/WHO Regional Office for Europe. Tuberculosis Surveillance and Monitoring in Europe. 2017. www.ecdc.europa.eu/sites/default/files/documents/ecdc-tuberculosis-surveillancemonitoring-Europe-2017-WEB.pdf

18 World Health Organization. Global Tuberculosis Report. 2019. www.who.int/tb/publications/global_report/en/

19 Scheurer D, Choudhry N, Swanton KA, et al. Association between different types of social support and medication adherence. Am J Manag Care 2012; 18: e461-e467.

20 Tennant R, Hiller L, Fishwick R, et al. The Warwick-Edinburgh Mental Well-being Scale (WEMWBS): development and UK validation. Health Qual Life Outcomes 2007; 5: 63.

21 WorldHealth Organization. Definitions and Reporting Framework for Tuberculosis - 2013 Revision. World Health Organization, 2013.

22 Sackett DL, Haynes RB. A critical review of determinants of patient compliance with therapeutic regimens. In: Compliance with Therapeutic Regimens. Baltimore, Johns Hopkins University Press, 1976; pp. 26-39. 